\title{
Safety and feasibility of rigid fixation by SternaLock Blu plates during the modified Ravitch procedure: a pilot study
}

\author{
Erik R. de Loos ${ }^{1}$, Paul C. M. Andel ${ }^{1}$, Jean H. T. Daemen ${ }^{1,2}$, Jos G. Maessen ${ }^{3,4}$, Karel W. E. Hulsewé ${ }^{1}$, \\ Yvonne L. J. Vissers ${ }^{1}$ \\ ${ }^{1}$ Department of Surgery, Division of General Thoracic Surgery, Zuyderland Medical Center, Heerlen, The Netherlands; ${ }^{2}$ Faculty of Health, \\ Medicine and Life Sciences (FHML), School for Oncology and Developmental Biology (GROW), Maastricht, The Netherlands; ${ }^{3}$ Department \\ of Cardiothoracic Surgery, Maastricht University Medical Center, Maastricht, The Netherlands; ${ }^{4}$ Faculty of Health, Medicine and Life Sciences \\ (FHML), Cardiovascular Research Institute Mastricht (CARIM), Maastricht, The Netherlands \\ Contributions: (I) Conception and design: ER de Loos, PCM Andel, JHT Daemen, YLJ Vissers; (II) Administrative support: KWE Hulsewé, JG \\ Maessen; (III) Provision of study materials or patients: ER de Loos, PCM Andel, JHT Daemen, YLJ Vissers; (IV) Collection and assembly of \\ data: YLJ Vissers, PCM Andel, JHT Daemen; (V) Data analysis and interpretation: ER de Loos, YLJ Vissers, KWE Hulsewé, JG Maessen; (VI) \\ Manuscript writing: All authors; (VII) Final approval of manuscript: All authors. \\ Correspondence to: Erik R. de Loos, MD. Department of Surgery, Division of General Thoracic Surgery, Zuyderland Medical Center, Henri \\ Dunantstraat 5, 6419 PC Heerlen, The Netherlands. Email: e.deloos@zuyderland.nl.
}

Background: Patients with anterior chest wall deformities unsuitable for minimally invasive repair are commonly treated by the modified Ravitch procedure. Although rigid plate fixation of the sternal osteotomy has previously shown to facilitate adequate sternal union, its use is troubled by an implant removal rate of up to $23 \%$ due to local complaints or complications associated with bulky plates. In contrast, the use of thinner and therefore biomechanically weaker plates may result in a higher incidence of non- or mal-union. In this pilot study, we evaluate the feasibility, efficacy and safety of rigid sternal fixation by thin pre-shaped anatomical locking plates during the modified Ravitch procedure.

Methods: Between June 2018 and December 2019, all consecutive patients who underwent anterior chest wall deformity repair by the modified Ravitch procedure in our tertiary referral centre were included. Data was collected retrospectively. All pectus types were included. The sternal osteotomy was fixated using thin SternaLock Blu plates. Patients were followed for at least one year.

Results: Nine patients were included. The group consisted of six male and three female patients, with a median age of 20 years [interquartile range (IQR), 16-35 years]. Median duration of follow-up was 25 months (IQR, 16-28 months). No intraoperative complications occurred. No patients presented with symptomatic non- or mal-union. Plate removal was performed in one patient for atypical pain without relief. No other postoperative complications occurred.

Conclusions: Based on these pilot results, thin SternaLock Blu plates are deemed to be safe and effective in providing adequate rigid fixation of the sternal osteotomy during the modified Ravitch procedure. Compared to literature, the need for plate removal within 25 months after surgery was reduced.

Keywords: Pectus; chest wall; modified Ravitch procedure; rigid sternal fixation; SternaLock Blu

Submitted Feb 16, 2021. Accepted for publication Apr 02, 2021.

doi: $10.21037 /$ jtd-21-284

View this article at: http://dx.doi.org/10.21037/jtd-21-284

(c) Journal of Thoracic Disease. All rights reserved. 


\section{Introduction}

Surgical treatment of congenital anterior chest wall deformities originated in the early 20th century and is, following adaptations, nowadays known as the modified Ravitch procedure (1). Pectus excavatum is the most common congenital anterior chest wall deformity and accounts for up to $90 \%$ of all deformities (2). Other less common anomalies include pectus carinatum and arcuatum (i.e., CurrarinoSilverman syndrome). During the modified Ravitch repair, the affected costal cartilage is resected while preserving the perichondrium, followed by correction of the sternal deformity through a cuneiform osteotomy. Although, less invasive surgical alternatives, such as the Nuss (for pectus excavatum), Abramson (for pectus carinatum) and sandwich (for pectus arcuatum) are currently considered as surgical treatment of first choice, the modified Ravitch procedure may still be indicated based on patient's age, severity or type of the deformity, prior thoracic surgery, experience of the surgical team and patient's preference (3).

In the modified Ravitch procedure, numerous methods and techniques have been proposed for sternal fixation after osteotomy, including cerclage or Kirschner wires, nonabsorbable meshes and struts (4). Yet, these only provide relative stability and are therefore associated with a risk of non-union (i.e., pseudoarthrosis), mal-union and postoperative pain. Symptomatic non-union is observed in up to $17 \%$ of patients undergoing the modified Ravitch procedure with a non-absorbable mesh (4). In addition, instability of the sternum reinforced by wire cerclages has been reported in $8 \%$ of cases after conventional cardiac surgery through a midsternal approach (5). Consequently, we have advocated for rigid sternal fixation through Locking Compression Plates (LCP) and previously reported on its efficacy in 26 consecutive patients after a modified Ravitch procedure. In this study we found no cases of symptomatic non- or mal-union after a mean follow-up of 30 months (4). Despite this apparent benefit, the disadvantage of the use of LCP is that they are bulky and may as a consequence be prominent underneath the thin sternal subcutaneous layer, necessitating hardware removal in 23\% of pectus patients (4). Kalberer et al. suggested an even higher LCP removal rate after osteosynthesis of sternal fractures (6). Implant removal is moreover associated with surgical wound infections in up to $12 \%$ of cases (7) and may even result in non-union if performed too early. Thinner and anatomical contoured locking plates are possibly effective in reducing the need for plate removal. SternaLock Blu (SternaLock Blu, Zimmer Biomet, Warsaw, Indiana, USA) is primarily used for fixation after median sternotomy with excellent outcomes $(8,9)$, and may therefore also be suitable for fixation of the sternum after correction of anterior chest wall deformities. However, until now, it is unknown whether the use of thinner, biomechanically weaker prevents the occurrence of non-and mal-union when used for fixation of the correction osteotomy in the modified Ravitch procedure.

The subsequent objective of this pilot study is to evaluate our preliminary single-center results regarding the feasibility, efficacy and safety of rigid sternal fixation by SternaLock Blu plates.

We present the following article in accordance with the STROBE reporting checklist (10) (available at http://dx.doi. org/10.21037/jtd-21-284).

\section{Methods}

\section{Study design}

A single-center retrospective observational pilot study was conducted. Prior to start, the study was approved by the local ethics and clinical research committee (Medical Ethics Review Committee Zuyderland, ID: METCZ20200049, approval date: June 8th, 2020), waiving the need for individual patient consent. The study was conducted in accordance with the Declaration of Helsinki (as revised in 2013).

\section{Participants}

All consecutive patients who underwent a modified Ravitch procedure for pectus excavatum, carinatum or arcuatum between June 2018 and December 2019 at Zuyderland Medical Center (Heerlen, The Netherlands) were included. In June 2018 we started using SternaLock Blu plates. December 2019 was chosen as end of the enrollment period to provide a minimum followup of one year. No distinction was made between pectus types since the common goal of the Ravitch procedure is to achieve an anatomical position of the sternum. Patients who were not eligible for minimally invasive repair by the Nuss (for pectus excavatum), Abramson (for pectus carinatum) or sandwich (for pectus arcuatum) procedure were included (e.g., due to the extreme severity of deformity, patient's age or preference). Patients with prior thoracic surgery were excluded.

\section{Preoperative evaluation, surgical technique and follow-up}

Patients were provided consultation at the outpatient clinic of our tertiary referral center for chest wall disorders. 

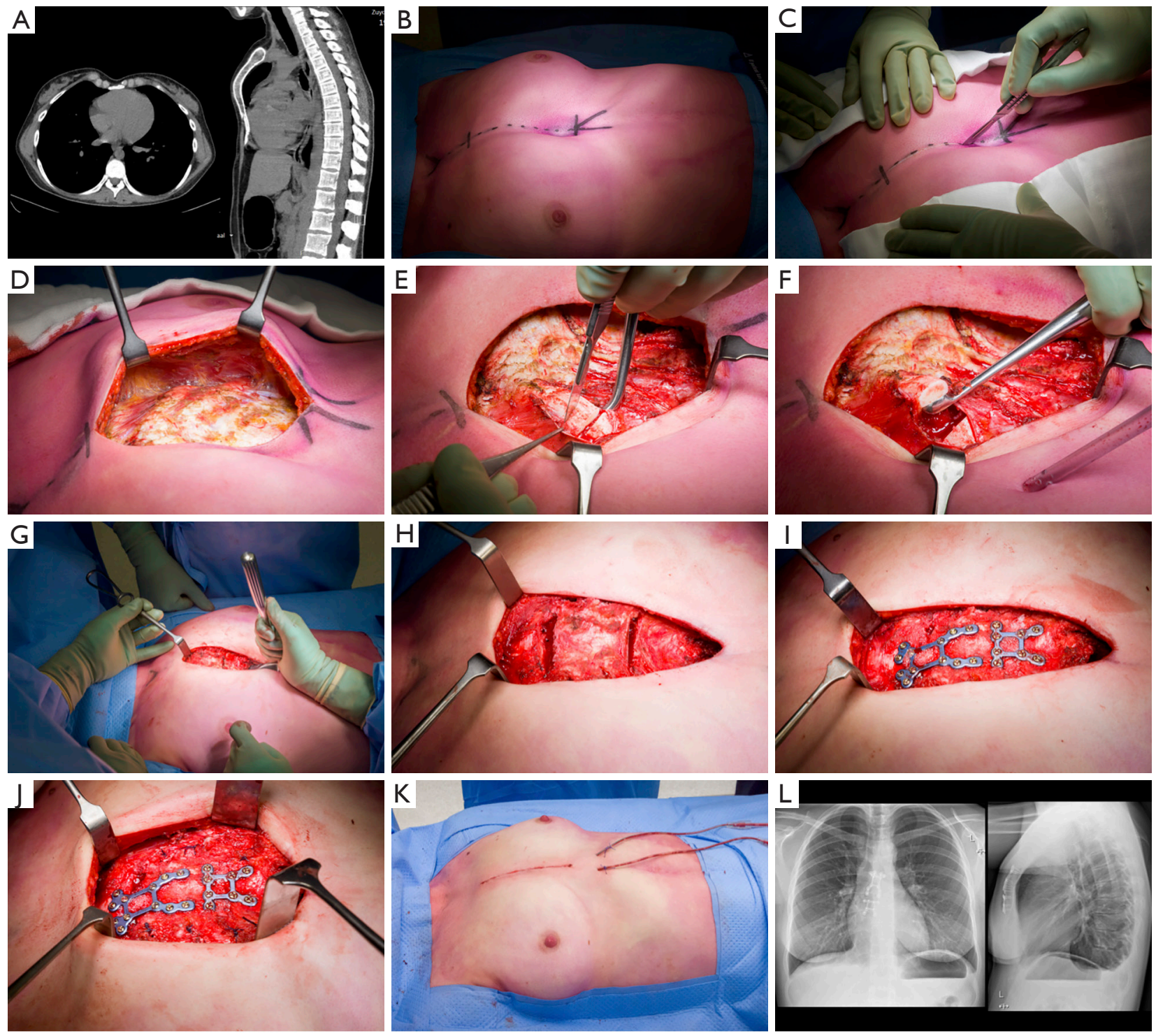

Figure 1 Surgical technique of the modified Ravitch procedure. (A) Preoperative evaluation by computed tomography. (B) Identification of the deepest point and incisional site. (C) Skin incision. (D) Bilateral pectoralis muscle dissection to expose the sternum and affected rib cartilage. (E,F) Resection of the deformed cartilage with preservation of the perichondrium. (G) Xiphoidectomy and sternal elevation. (H) Sternal osteotomies. (I) Fixation of the sternal osteotomies by the SternaLock Blu system. (J) Approximation of the perichondrium. (K) Placement of low-vacuum drains and closure in layers. (L) Postoperative result.

Preoperative evaluation included a chest computed tomography (CT) scan to evaluate the severity of the deformity (Figure 1A). For patients suffering from pectus excavatum, the Haller index was determined, and the presence of cardiac compression evaluated. Additional assessment of cardiorespiratory function was performed upon indication.

Surgery was performed under epidural and general anesthesia with a single-lumen endotracheal tube. The patient was positioned supine and prophylactic cefazolin was administered. Following skin markings and identification of the deepest point (Figure 1B), a midline skin incision was made over the sternal deformity (Figure 1C). The pectoralis muscles were bilaterally dissected to expose the sternum and affected rib cartilage (Figure 1D). All deformed cartilage segments were resected 
while preserving the perichondrium (Figure $1 E, F$ ). A xiphoidectomy was performed in order to lift the sternum and avoid postoperative prominence (Figure 1G). Subsequently, a wedge osteotomy was made at the cranial margin of the sternal deformity using an osteotome. If necessary, to achieve a neutral sternal position, a second osteotomy was performed (Figure $1 H$ ). The wedge cuts were made in such fashion to facilitate optimal bone contact between the separated surfaces and allow proper sternal union. The SternaLock Blu system (SternaLock Blu, Zimmer Biomet, Warsaw, Indiana, USA) was used for fixation of the sternal osteotomy with plates and selfdrilling locking screws (Figure 1I). No additional support methods were employed (e.g., rib plates or retrosternal support by struts). After fixation, the perichondrium was approximated (Figure 17), and bilateral low-vacuum drains were placed. The pectoralis major muscles, subcutaneous tissue and skin were closed in layers (Figure $1 \mathrm{~K}$ ). A postoperative anteroposterior plain radiograph was acquired to rule out the presence of a pneumothorax (Figure $1 L$ ). In addition, a lateral plain radiograph was obtained during the first postoperative days in order to evaluate the postoperative result (Figure 1L). Patients were discharged following drain removal (less than $35 \mathrm{cc}$ per 24 hours), adequate pain control and mobilization. Standard follow-up consisted of an outpatient clinic visit after two weeks. Additional visits were scheduled on demand through shared decision making.

\section{Variables and data acquisition}

Patient charts were retrospectively reviewed for (I) baseline patient characteristics (sex, age, type of deformity and for pectus excavatum the Haller index and presence or absence of cardiac compression), (II) operation characteristics (duration, blood loss and intraoperative complications) and (III) postoperative characteristics [length of hospital stay, complications (graded by the Clavien-Dindo classification) (11) and length of followup]. Symptomatic non-union was defined as the presence of symptoms (i.e., pain or subjective instability) in combination with a CT confirmed non-union at least 6 months after surgery. Implant removal due to complaints (e.g., pain or prominence) was only acknowledged as such, if symptoms resolved after removal.

\section{Statistical analyses}

Statistical analyses were performed using SPSS Statistics
(IBM Corp. IBM SPSS Statistics for MacOS, Version 27.0, Armonk, New York, USA). Continuous variables were depicted as median, interquartile range (IQR) and range. Categorical variables were denoted as frequencies and percentages. Missing data was reported as such.

\section{Results}

Between June 2018 and December 2019, nine consecutive patients underwent a modified Ravitch procedure with application of the SternaLock Blu system. Six out of nine patients were male $(67 \%)$ with a median age of 20 years (IQR, 16-35; range, 13-59 years). The group consisted of four patients $(45 \%)$ with pectus excavatum, three $(33 \%)$ with pectus carinatum and two $(22 \%)$ with pectus arcuatum. The indication to perform a modified Ravitch procedure instead of a minimally invasive approach was age in 2 out of 4 pectus excavatum patients and extreme severity in the remaining 2. In addition, reasoning for an open approach encompassed a combination of severity and potential inability to redress the deformity by the Abramson method in all pectus carinatum patients while the modified Ravitch procedure was favored due to age in all pectus arcuatum patients. The Haller index among patients with pectus excavatum ranged from 3.0-4.9, compared to 2.2-2.9 ( $\mathrm{n}=1$ missing) after surgery. The median length of surgery was 104 minutes (IQR, 99-142; range, 89-171 minutes) with a median blood loss of $50 \mathrm{~mL}$ (IQR, 35-200; range, 20-300 mL). No intra-operative complications occurred. Postoperative lateral plain radiographs demonstrated an anatomical position of the sternum in all patients $(n=1$ missing). Patients were discharged after a median of 5 days (IQR, 5-7; range, 4-8 days). Baseline patient characteristics are summarized in Table 1.

One-year follow-up was available for all patients. Median duration of follow-up was 25 months (IQR, 16-28; range, 15-29 months). No patients presented with symptomatic non-union or mal-union. In addition, no recurrent cases were observed. In one 46-year-old male patient, plates were removed due to atypical pain complaints (CDC-IIIb). However, symptoms did not improve upon removal, making an association with the osteosynthesis materials unlikely. Additional cardiac and pulmonary evaluation did not reveal any abnormalities and symptoms were classified as idiopathic. No secondary surgical or other complications occurred. Peri- and postoperative outcomes are summarized in Table 2. 
Table 1 Baseline patient characteristics

\begin{tabular}{lccc}
\hline \multirow{2}{*}{ Characteristics } & \multicolumn{3}{c}{ SternaLock Blu (n=9) } \\
\cline { 2 - 4 } & Median or $\mathrm{n}$ [\%] & IQR & Range \\
\hline Age (years) & $6[67]$ & $16-35$ & $13-59$ \\
Male & & & \\
Pectus type & $4[45]$ & & \\
Pectus excavatum & $3[33]$ & \\
Pectus carinatum & $2[22]$ & $3.0-4.9$ \\
Pectus arcuatum & & & \\
Haller index & & & \\
\hline
\end{tabular}

$\mathrm{n}$, number; IQR, interquartile range.

\section{Discussion}

The aim of this pilot study was to evaluate the feasibility, efficacy and safety of rigid sternal fixation by the SternaLock Blu system during the modified Ravitch procedure. We started using these thinner, pre-shaped anatomical locking plates in June 2018 because of the relatively high plate removal rate of $23 \%$ using LCP in an earlier cohort (4). This high removal rate was attributed to the bulkiness and subsequent prominence of LCP, causing complaints. This is in accordance with a series reporting a plate removal rate of $27 \%$ in fifteen patients who received locking compression plate fixation after sternotomy with a mean follow-up of 57 months (6).

Using SternaLock Blu plates in the current series, the hardware was removed in only one patient suffering from thoracic pain. However, because his symptoms persisted afterwards and no physical substrate was found by extensive cardiopulmonary evaluation, the pain was concluded not to be plate related. This emphasizes the need for careful patient selection before advancing to plate removal. In addition, if plate removal is indicated based on plate prominence, removal is advised to be postponed until adequate union is likely (at least 6 months after initial surgery) and moreover confirmed by cross-sectional imaging.

Based on the comparison with available literature, the thinner SternaLock plates seem to be associated with reduced need for removal due to plate prominence and associated burden compared to the bulkier LCP. In turn, this may prevent secondary surgical complications such as non-union and surgical site infections following removal (7). However, it has to be noted that the follow-up of both LCP groups was considerably longer (respectively 30 and 57 months) than the SternaLock Blu group from the current series
Table 2 Peri- and postoperative outcomes

\begin{tabular}{|c|c|c|c|}
\hline \multirow{2}{*}{ Characteristics } & \multicolumn{3}{|c|}{ SternaLock Blu $(n=9)$} \\
\hline & Median or $\mathrm{n}[\%]$ & IQR & Range \\
\hline Length of surgery (minutes) & 104 & $99-142$ & $89-171$ \\
\hline $\begin{array}{l}\text { Blood loss during surgery } \\
(\mathrm{mL})\end{array}$ & 50 & $35-200$ & $20-300$ \\
\hline Hospital stay (days) & 5 & $5-7$ & $4-8$ \\
\hline \multicolumn{4}{|l|}{ NRS } \\
\hline Postoperative & 2 & $0-5$ & $0-10$ \\
\hline Discharge & 2 & $0-3$ & $0-3$ \\
\hline Follow-up (months) & 25 & $16-28$ & $15-29$ \\
\hline Postoperative complications & $0[0]$ & & \\
\hline Seroma & 0 & & \\
\hline Wound dehiscence & 0 & & \\
\hline Wound infection & 0 & & \\
\hline Pectus recurrence & 0 & & \\
\hline Re-admission & 0 & & \\
\hline Non-union & 0 & & \\
\hline Mal-union & 0 & & \\
\hline Plate removal & $1[11]$ & & \\
\hline Pain & $1^{*}$ & & \\
\hline Plate prominence & 0 & & \\
\hline
\end{tabular}

*, plate removal had no effect on pain. IQR, Interquartile range; $\mathrm{SD}$, standard deviation; $\mathrm{mL}$, milliliters; NRS, Numeric Rating Scale.

(median: 25 months).

Since publications on the use of sternal plates in Ravitch procedures are limited to the previously mentioned report by our center, we also reviewed literature on the use of SternaLock Blu plates after cardiac sternotomy. In a study by Raman and colleagues (8), a hardware removal rate of $11 \%(n=8 / 70)$ was found. Of these 8 plate removals, 6 were removed within seven to 82 days after surgery due to screw back out, pull through, wound infection and a non-infected wound sinus. The median follow-up of our current study was long enough (i.e., median follow up of 25 months) to observe these complications, however, none occurred. Nevertheless, all patients studied by Raman $e t a l$. were preoperatively determined to be at high risk for sternal wound complications. In contrast, the pectus population is generally younger with fewer comorbidities than patients undergoing cardiac surgery (8). 
In this study, we did not routinely acquire postoperative CT scans to assess the presence of non- or mal-union cases. We solely assessed symptomatic (e.g., pain, discomfort or hardware failure) cases by means of CT since asymptomatic cases do generally not require re-intervention. Though, we acknowledge that this may lead to a potential underestimation of the true occurrence of non- and malunion. In the present series, no cases of symptomatic nonunion or mal-union were detected during the median follow-up of 25 months. This is comparable to our previous experience in 26 pectus excavatum patients treated with locking compression plate fixation as part of a modified Ravitch procedure, where no cases of symptomatic nonor mal-union were detected after a mean follow-up of 30 months (4). We therefore suggest the thinner SternaLock Blu plates to be an effective alternative for sternal fixation, providing sufficient rigidity as demonstrated by the absence of symptomatic non-union and mal-union cases. However, it should be noted that the risks on plate failure differ among pectus types. Especially pectus excavatum is theoretically at higher risk for failure.

Based on a large series $(\mathrm{n}=426)$ by Masaoka et al., complications during the modified Ravitch procedure occur in approximately $6 \%$ of cases (12). Potential complications are primarily wound- (i.e., seroma and wound dehiscence) and respiratory-related (i.e., pneumothorax, pneumonia, pleural effusion and atelectasis). Masaoka et al. created a bridge consisting of resected costal cartilage to support the sternum, thus performing a metal-free Ravitch procedure, while Fonkalsrud in a series of 912 patients with metal strut support revealed a complication rate of $8 \%$ (13). In comparison, in a smaller series using cerclage wires with non-absorbable retrosternal mesh support $(\mathrm{n}=18)$ or LCP $(n=26)$, a complication rate (other than hardware removal and non-union) of respectively $33 \%$ and $15 \%$ is described (4). In the current series, using SternaLock Blu plates for rigid fixation of the sternal osteotomy among nine patients, no complications were observed.

\section{Limitations}

To the best of our knowledge, the current study is the first to investigate the use of SternaLock Blu plates for rigid sternal fixation during the modified Ravitch procedure. Nonetheless, this study is subject to limitations. Retrospective cohort studies are inherently susceptible to bias due to missing data. Yet, only one postoperative lateral radiograph was missing, as a result of which the postoperative Haller index could not be determined in one patient. In addition, it should be noted that the study's findings concern pilot data obtained during adaption of the use of SternaLock Blu plates. Moreover, conducting a pilot study is inherently associated with a small sample size, limiting evidential value. In addition, potential underestimation of asymptomatic non- and mal-union cases is possible, given that cross-sectional imaging was only performed in the presence of symptoms.

Currently, more minimally invasive procedures, such as the Nuss procedure, are favored over the modified Ravitch procedure due to smaller incisions, less blood loss and shorter operative time (14). Likewise, a minimally invasive treatment (i.e., the Abramson procedure) or conservative treatment (i.e., bracing or dynamic compression therapy) is favored for pectus carinatum (15). Since the common goal has been to minimize surgical trauma, the modified Ravitch procedure has gradually declined in relative incidence, as also demonstrated by Zuidema et al. (16). This may have led to selection bias given that, in particular, more severe cases are selected for the modified Ravitch procedure. This explains the small and heterogenous study population of this study which may limit extrapolation of results to other patient cohorts. Future comparative research should focus to expand and strengthen the current evidence with adequate power.

\section{Conclusions}

Based on our first experience, SternaLock Blu plates are suggested to be a safe and feasible method for rigid sternal fixation during the modified Ravitch procedure. Moreover, in the absence of non-union and mal-union cases, they are suggested to provide adequate sternal stability. Furthermore, SternaLock Blu plates are potentially associated with reduced need for hardware removal compared to bulkier LCP. Future comparative studies are needed to strengthen the evidence.

\section{Acknowledgments}

Funding: None.

\section{Footnote}

Reporting Checklist: The authors have completed the STROBE reporting checklist. Available at http://dx.doi. org/10.21037/jtd-21-284 
Data Sharing Statement: Available at http://dx.doi. org/10.21037/jtd-21-284

Peer Review File: Available at http://dx.doi.org/10.21037/jtd21-284

Conflicts of Interest: All authors have completed the ICMJE uniform disclosure form (available at http://dx.doi. org/10.21037/jtd-21-284). The authors have no conflicts of interest to declare.

Ethical Statement: The authors are accountable for all aspects of the work in ensuring that questions related to the accuracy or integrity of any part of the work are appropriately investigated and resolved. The study was conducted in accordance with the Declaration of Helsinki (as revised in 2013). The study was approved by the local ethics and clinical research committee (Medical Ethics Review Committee Zuyderland, ID: METCZ20200049, approval date: June 8th, 2020), waiving the need for individual patient consent.

Open Access Statement: This is an Open Access article distributed in accordance with the Creative Commons Attribution-NonCommercial-NoDerivs 4.0 International License (CC BY-NC-ND 4.0), which permits the noncommercial replication and distribution of the article with the strict proviso that no changes or edits are made and the original work is properly cited (including links to both the formal publication through the relevant DOI and the license). See: https://creativecommons.org/licenses/by-nc-nd/4.0/.

\section{References}

1. Ravitch MM. The Operative Treatment of Pectus Excavatum. Ann Surg 1949;129:429-44.

2. Fokin AA, Steuerwald NM, Ahrens WA, et al. Anatomical, histologic, and genetic characteristics of congenital chest wall deformities. Semin Thorac Cardiovasc Surg 2009;21:44-57.

3. Erşen E, Demirkaya A, Kılıç B, et al. Minimally invasive repair of pectus excavatum (MIRPE) in adults: is it a proper choice? Wideochir Inne Tech Maloinwazyjne 2016;11:98-104.

4. de Loos ER, Hulsewé KWE, van Loo ERJ, et al. Does the use of locking plates or mesh and wires influence the risk of symptomatic non-union of the sternal osteotomy after modified Ravitch? J Thorac Dis 2020;12:3631-9.
5. Robicsek F, Fokin A, Cook J, et al. Sternal instability after midline sternotomy. Thorac Cardiovasc Surg 2000;48:1-8.

6. Kalberer N, Frima H, Michelitsch C, et al. Osteosynthesis of sternal fractures with double locking compression plate fixation: a retrospective cohort study. Eur J Orthop Surg Traumatol 2020;30:75-81.

7. Backes M, Schep NW, Luitse JS, et al. High Rates of Postoperative Wound Infection Following Elective Implant Removal. Open OrthopJ 2015;9:418-21.

8. Raman J, Lehmann S, Zehr K, et al. Sternal closure with rigid plate fixation versus wire closure: a randomized controlled multicenter trial. Ann Thorac Surg 2012;94:1854-61.

9. Allen KB, Thourani VH, Naka Y, et al. Randomized, multicenter trial comparing sternotomy closure with rigid plate fixation to wire cerclage. J Thorac Cardiovasc Surg 2017;153:888-896.e1.

10. von Elm E, Altman DG, Egger M, et al. The Strengthening the Reporting of Observational Studies in Epidemiology (STROBE) statement: guidelines for reporting observational studies. J Clin Epidemiol 2008;61:344-9.

11. Dindo D, Demartines N, Clavien PA. Classification of surgical complications: a new proposal with evaluation in a cohort of 6336 patients and results of a survey. Ann Surg 2004;240:205-13.

12. Masaoka A, Kondo S, Sasaki S, et al. Thirty years' experience of open-repair surgery for pectus excavatum: development of a metal-free procedure. Eur J Cardiothorac Surg 2012;41:329-34.

13. Fonkalsrud EW. 912 open pectus excavatum repairs: changing trends, lessons learned: one surgeon's experience. World J Surg 2009;33:180-90.

14. Mao YZ, Tang S, Li S. Comparison of the Nuss versus Ravitch procedure for pectus excavatum repair: an updated metaanalysis. J Pediatr Surg 2017;52:1545-52.

15. Emil S. Current Options for the Treatment of Pectus Carinatum: When to Brace and When to Operate? Eur J Pediatr Surg 2018;28:347-54.

16. Zuidema WP, van der Steeg AFW, Oosterhuis JWA, et al. Trends in the Treatment of Pectus Excavatum in the Netherlands. Eur J Pediatr Surg 2020. doi: 10.1055/s-0040-1712182.

Cite this article as: de Loos ER, Andel PCM, Daemen JHT, Maessen JG, Hulsewé KWE, Vissers YLJ. Safety and feasibility of rigid fixation by SternaLock Blu plates during the modified Ravitch procedure: a pilot study. J Thorac Dis 2021;13(5):29522958. doi: $10.21037 / \mathrm{jtd}-21-284$ 